\title{
The Impact of Emergency Department Observation Units on United States Emergency Department Admission Rates
}

\author{
Roberta Capp, MD, MHS ${ }^{1 \star}$, Benjamin Sun, MD, MPH², Dowin Boatright, MD, MBA ${ }^{3}$, Cary Gross, MD, $\mathrm{MPH}^{4}$
}

\begin{abstract}
${ }^{1}$ Department of Emergency Medicine, University of Colorado School of Medicine, Aurora, Colorado; ${ }^{2}$ Department of Emergency Medicine, Oregon Health and Science University, Portland, Oregon; ${ }^{3}$ Department of Emergency Medicine, Denver Health Medical Center, Denver, Colorado; ${ }^{4}$ Robert Wood Johnson Foundation Clinical Scholars Program, Section of Internal Medicine, Yale University School of Medicine, New Haven, Connecticut.
\end{abstract}

OBJECTIVE: Prior studies suggesting that the presence of emergency department (ED) observation units decrease overall ED hospital admissions have been either singlecenter studies or based on model simulations. The objective of this preliminary national study is to determine if the presence of ED observation units is associated with hospitals having lower ED admission rates.

METHODS: We conducted a retrospective cross-sectional analysis using the 2010 National Hospital Ambulatory Care Survey and estimated ED risk-standardized hospital admission rates (RSHAR) for each center. The following were excluded from the study: ages $<18$ years, leaving prior to completion of ED visit, died in the ED, transferred to another facility, and missing disposition. Hospitals with less than 30 ED visits or unknown observation unit status were also excluded. We used linear regression analysis to determine the association between ED RSHAR and presence of observation units.
RESULTS: There were 24,232 ED visits in 315 hospitals in the United States. Of these, $82(20.6 \%)$ hospitals had an ED observation unit. The average ED risk-standardized hospital admission rates for hospitals with observation units and without hospital observation units were $13.7 \%$ (95\% confidence interval [Cl]: 11.3-16.0) and 16.0\% (95\% Cl: $14.1-$ $17.7)$, respectively. The difference of $2.3 \%$ was not statistically significant.

CONCLUSIONS: In this preliminary study, we did not find an association between the presence of observation units and ED hospital admission rates. Further studies with larger sample sizes should be performed to further evaluate the impact of ED observation units on ED hospital admission rates. Journal of Hospital Medicine 2015;10:738-742. (C) 2015 Society of Hospital Medicine
Today more than one-third of emergency departments (EDs) in the United States have affiliated observation units, where patients can stay 24 to 48 hours without being admitted to the hospital. ${ }^{1}$ Observation units experienced significant growth in the United States from 2005 to 2007, secondary to policy changes involving the Centers for Medicare and Medicaid Services (CMS), which expanded reimbursement for observation services to include any clinical condition. Furthermore, CMS implemented the Recovery Audit Contractor process, which was able to fine providers and facilities for inappropriate claims, with the principle method for charge recovery being "inappropriate" charges for short inpatient stays.

ED observation units (EDOUs) vary in the number of beds, but are often located adjacent to the emergency department. ${ }^{2}$ It is estimated that EDOUs have

*Address for correspondence and reprint requests: Roberta Capp, MD, Department of Emergency Medicine, University of Colorado School of Medicine, Aurora, CO 80045; Telephone: 720-848-4270; Fax: 720-8487374;

E-mail: Roberta.Capp@ucdenver.edu

Additional Supporting Information may be found in the online version of this article.

Received: March 18, 2015; Revised: July 9, 2015; Accepted: July 22, 2015

2015 Society of Hospital Medicine DOI 10.1002/jhm.2447

Published online in Wiley Online Library (Wileyonlinelibrary.com). the capacity for caring for $5 \%$ to $10 \%$ of any given ED volume. ${ }^{2}$ Almost half of EDOUs are protocol driven, allowing these units to discharge up to $80 \%$ of all patients within 24 hours. ${ }^{1,2}$ Some studies have suggested that EDOUs are associated with a decrease in overall hospitalization rates, leading to cost savings. ${ }^{1}$ However, these studies were limited by their single-center design or simulated in nature. In addition, other studies show that EDOUs decrease inpatient admissions, length of stay, and costs related to specific clinical conditions such as chest pain, transient ischemic attack, and syncope. ${ }^{3}$

To further evaluate the association of observation units on ED hospital admission rates nationally, we analyzed the largest ED-based survey, the 2010 National Hospital Ambulatory Medical Care Survey (NHAMCS), to assess the impact of observation units on hospital admissions from the ED. We hypothesized that observation units decrease overall hospital admissions from the ED.

\section{METHODS}

\section{Study Design and Population}

We performed a retrospective cross-sectional analysis of ED visits from 2010. This study was exempt from institutional review board review by the University of Colorado and Yale University institutional review committee. The NHAMCS is an annual, national 
probability sample of ambulatory visits made to nonfederal, general, and short-stay hospitals conducted by the Centers for Disease Control and Prevention (CDC), National Center for Health Statistics. The multistaged sample design was previously described elsewhere. ${ }^{4}$ The 2010 NHAMCS dataset included 350 participating hospitals (unweighted sampling rate of $90 \%$ ) and a total of 34,936 patient visits. ${ }^{4}$

\section{Exclusions}

We excluded patients who were less than 18 years old ( $\mathrm{n}=8015 ; 23 \%)$; left without being seen, left before examination completion, or left against medical advice $(\mathrm{n}=813 ; 2 \%)$; transferred to another institution $(\mathrm{n}=$ $626 ; 1.7 \%)$; died on arrival or died in the ED $(\mathrm{n}=$ $60 ; 0.2 \%)$; and with missing data on discharge disposition $(\mathrm{n}=100 ; 0.3 \%)$. Finally, we excluded hospitals with fewer than 30 visits per year $(\mathrm{n}=307 ; 0.9 \%)$ to comply with reliable relative standard errors, as recommended by the CDC; after all of these exclusions there were 325 hospitals. Finally, we excluded hospitals with missing information on EDOUs ( $\mathrm{n}=783$, $2.2 \%)$; our dataset at this point included 315 hospitals.

\section{Outcomes}

The primary outcome was hospital admission, either from the ED or admitted to an observation unit with subsequent hospital admission, defined as the ED riskstandardized hospital admission rate (ED RSHAR). ${ }^{5}$ This methodology allows for risk adjustment of case mix (ie, disease severity) for each hospital's ED admission rates and has been previously described in the evaluation of varying ED hospital admission rates using the same dataset. ${ }^{5}$ To evaluate which hospitals had observation units, we used the following hospital survey question: "Does your ED have an observation or clinical decision unit?"

\section{Identification of Variables}

ED hospitalization rates were risk standardized for each hospital to account for each hospital's case mix and hospital factors such as socioeconomic status, clinical severity, and hospital characteristics. This methodology and dataset use have been previously described in detail. ${ }^{5}$

To account for common chief complaints leading to hospitalization and case-mix distribution of these complaints among different hospitals, we analyzed all chief complaints and their relationship to hospital admission. We first identified those associated with an admission rate that exceeded $30 \%$ and was present in $1 \%$ or more of patient visits. The study team of researchers and clinicians determined the aforementioned cutoffs as clinically meaningful. Eight chief complaints met both criteria: chest pain and related symptoms, shortness of breath, other symptoms/probably related to psychological, general weakness, labored or difficulty breathing, fainting (syncope), unconscious arrival, and other symptoms referable to the nervous system. Chronic diseases, such as congestive heart failure, diabetes mellitus, renal disease on dialysis, and human immunodeficiency virus, were also included in the model.

Hospital factors included metropolitan status, geographic region of the country (limited to Northeast, Midwest, South, and West), teaching status, and urban or rural status. ${ }^{6}$ We derived a new variable based on a previous study, teaching status, by combining nonprivate hospital status plus having at least 1 ED visit be evaluated by a resident.

\section{Statistical Analyses}

We used SAS version 9.2 (SAS Institute, Cary, NC) for all statistical analyses. Frequencies of all variables in the model were calculated to assess the distribution of data and quantify missing data. We did not want to have variables in the model with high collinearity. To investigate collinearity between independent variables, we calculated Spearman correlation coefficients; high collinearity was defined as $r>0.6$. No variables included in the model had high collinearity.

To investigate the association of the candidate variables with hospitalization, we used survey logistic regression. Although some variables did not show an association with hospitalization, we felt they were clinically relevant and did not remove them from the model. Hierarchical logistic regression modeling (explained below) was used to calculate ED RSHAR based on the aforementioned selected variables associated with hospital admission.

Hierarchical logistic regression models (HLRM) were used to estimate RSHAR for each hospital. This approach reflects the assumption that a hospitalspecific component exists, and that it will affect the outcomes of patients at a particular institution. This method takes into consideration the hierarchical structure of the data to account for patient clustering within hospitals, and has been used by the CMS to publicly report hospital risk-standardized rates of mortality and readmission for acute myocardial infarction, heart failure, and pneumonia.

We used a similar methodology as previously published..$^{5}$ In summary, the hospital RSHAR was calculated as a ratio of the number of predicted hospital admissions in the hospital to the number of expected hospital admissions in the hospital. This ratio is then multiplied by the national unadjusted rate of hospital admissions. We calculated the $\mathrm{C}$ statistic of the HLRM model to assess for overall adequacy of risk prediction. To analyze the association between ED RSHAR and EDOUs, we used analysis of variance, where the dependent variable was ED RSHAR and independent variable of interest was presence of EDOUs. 


\begin{tabular}{|c|c|c|c|}
\hline & $\begin{array}{c}\text { Hospitals With } \\
\text { Observation Units, } \\
\text { W\% }(\mathrm{N}=82)\end{array}$ & $\begin{array}{l}\text { Hospitals Without } \\
\text { Observation Units, } \\
\text { W\% ( }=233)\end{array}$ & $P$ Value \\
\hline Region of country & & & 0.54 \\
\hline Northeast & 10.01 & 15.46 & \\
\hline Midwest & 32.06 & 28.35 & \\
\hline South & 41.84 & 36.33 & \\
\hline West & 16.08 & 19.85 & \\
\hline Ownership of hospitals & & & 0.4 \\
\hline Voluntary, nonprofit & 77.28 & 72.35 & \\
\hline Government, nonfederal & 18.78 & 16.11 & \\
\hline Private & 3.94 & 11.55 & \\
\hline Urban or rural location & & & 0.43 \\
\hline Urban & 68.28 & 60.19 & \\
\hline Rural & 31.72 & 39.81 & \\
\hline Teaching hospital status & & & 0.56 \\
\hline Teaching hospital & 63.22 & 68.28 & \\
\hline Nonteaching hospital & 36.78 & 31.71 & \\
\hline
\end{tabular}

\section{RESULTS}

There were 24,232 ED visits from 315 hospitals in the United States in our study. Of these, 82 (20.6\%) hospitals had an observation unit physically separate from the ED. Hospitals with and without observation units did not have different hospital patient level characteristics. There was no association between hospital ownership, teaching status, region location, urban or rural location, and hospitals with observation units when compared with hospitals without observation units (Table 1).

In addition, there was no association between patient characteristics at the ED visit level in hospitals with observation units when compared with patient characteristics at the ED visit level in hospitals without observation units (Table 2). The average ED riskstandardized hospital admission rate for hospitals with observation units was $13.7 \%$ (95\% confidence interval [CI]: 11.3 to 16.0 ) compared to $16.0 \%$ (95\% CI: 14.1 to 17.7$)$ for hospitals without observation units (Figure 1). This difference of $2.3 \% \quad(95 \%$ CI: -0.1 to 4.7 ) was not statistically significant.

\section{DISCUSSION}

In this national study of hospital admissions from the ED, we did not find that hospitals with observation units had a statistically significant lower ED riskstandardized admission rate when compared with hospitals that did not have observation units. However, the difference of ED risk-standardized hospital admission rates between hospitals with observation units and those without observation units was very small, and we were likely underpowered to detect a statistically significant difference.

Recently, EDOUs have received much attention, in part because of increases in their numbers and frequency of use. ${ }^{7}$ Prior studies, which did not report admission rates that were risk standardized, have also demonstrated no difference in the admission rates among hospitals with and without observation units. ${ }^{6,8}$ Although this result seems counterintuitive, several possible explanations exist.

One reason that there may not be a relation between the rate of inpatient admission and the presence of an observation unit is that the introduction of an EDOU appears to change physician behavior. When the option to admit to an observation unit is present, ED physicians are 2 times more likely to disposition patients to observation status without a statistically significant change in the rate of inpatient admission. ${ }^{6}$ Studies have demonstrated that after the introduction of an observation unit, ED physicians tend to overutilize observation among patients who previously would have been discharged, while continuing to admit patients as inpatients who meet observation criteria, which could result in an increase in cost for payers and patients. ${ }^{7,9}$

Observation units that are protocol driven have been associated with the best patient outcomes including shorter length of stay, lower likelihood of subsequent inpatient admission, and decreased cost. ${ }^{10}$ Furthermore, studies evaluating EDOUs suggest increased patient satisfaction and improved patient safety, especially for protocol-driven EDOUs. ${ }^{2}$ However, currently, only half of dedicated observation units are protocol driven. It is also possible that the ED inpatient admission rate does not capture the full impact of an observation unit on care delivery and quality. Observation units are more likely to be present in EDs with a higher overall patient census, longer patient lengths of stay, and higher rates of ambulance diversion. ${ }^{6,8}$ Unfortunately, NHAMCS does not distinguish protocol-driven versus non-protocol-driven observation units. From a policy standpoint, as EDOUs continue to emerge, there is an opportunity to standardize how EDOUs function by using best practices.

This study should be evaluated in the context of limitations such as heterogeneity in the management of EDOUs, limited hospital factor variables that may influence hospital admissions, and small sample size associated with each hospital. Because we were not able to determine which EDs used protocol-driven observation units, we were not able to determine the impact of having a protocol-driven observation unit on inpatient hospital admission rates. Additionally, the study may suffer from a selection bias, as EDs with observation units have been shown to have higher patient volume, longer patient lengths of stay, and greater rates of ED diversion. Despite the small sample size, our risk-standardized model accounted for case mix and hospital factors associated with hospital admission rates and had a high $\mathrm{C}$ statistic value, which indicates that the predicted probability of being admitted from the ED highly correlates with the actual outcome of being admitted from the ED. We 
TABLE 2. Emergency Department Patient Level Characteristics in Hospitals With and Without Observations Units

\begin{tabular}{|c|c|c|c|}
\hline & $\begin{array}{c}\text { Hospitals With } \\
\text { Observation Units, } \\
\text { W\% }(\mathrm{N}=6,067)\end{array}$ & $\begin{array}{l}\text { Hospitals Without } \\
\text { Observation Units, } \\
\text { W\% (N=18,165) }\end{array}$ & $P$ Value \\
\hline Sex, female & 58.75 & 58.35 & 0.96 \\
\hline Age, y & 45.17 & 46.08 & 0.32 \\
\hline Race & & & 0.75 \\
\hline Non-Hispanic white & 63.54 & 66.41 & \\
\hline Non-Hispanic black & 23.67 & 18.77 & \\
\hline Hispanic & 9.77 & 12.47 & \\
\hline Other & 3.02 & 2.35 & \\
\hline Source of payment & & & 0.87 \\
\hline Private & 21.90 & 21.46 & \\
\hline Medicare & 32.73 & 30.55 & \\
\hline Medicaid & 22.15 & 23.23 & \\
\hline Uninsured & 18.61 & 20.25 & \\
\hline Unknown/missing & 4.61 & 4.51 & \\
\hline Poverty level & & & 0.50 \\
\hline$<5 \%$ & 13.87 & 15.31 & \\
\hline $5 \%-9.9 \%$ & 32.57 & 23.38 & \\
\hline $10 \%-19.9 \%$ & 29.81 & 36.29 & \\
\hline$>20 \%$ & 20.32 & 20.18 & \\
\hline Missing & 3.44 & 4.83 & \\
\hline Arrival by ambulance & & & 0.06 \\
\hline Yes & 20.01 & 18.61 & \\
\hline No & 76.12 & 76.34 & \\
\hline Unknown & 3.87 & 5.05 & \\
\hline Severity of illness & & & 0.58 \\
\hline Emergent & 16.58 & 16.62 & \\
\hline Nonemergent & 44.09 & 43.85 & \\
\hline Indeterminate & 1.18 & 1.17 & \\
\hline Mental health, alcohol, unclassified & 38.15 & 38.37 & \\
\hline \multicolumn{4}{|l|}{ Vital signs } \\
\hline Temperature & & & 0.91 \\
\hline $90^{\circ}-95^{\circ} \mathrm{F}$ & 0.31 & 0.36 & \\
\hline $95.1^{\circ}-100.4^{\circ} \mathrm{F}$ & 93.94 & 93.19 & \\
\hline $100.4^{\circ}-107^{\circ} \mathrm{F}$ & 1.81 & 2.11 & \\
\hline Missing & 3.94 & 4.35 & \\
\hline Pulse & & & 0.60 \\
\hline 10-59 bpm & 3.39 & 3.93 & \\
\hline $60-100 \mathrm{bpm}$ & 72.86 & 75.94 & \\
\hline$>101$ bpm & 19.60 & 21.37 & \\
\hline Missing & 4.16 & 7.67 & \\
\hline Systolic blood pressure & & & 0.92 \\
\hline $50-90 \mathrm{~mm} \mathrm{Hg}$ & 0.90 & 1.02 & \\
\hline $91-160 \mathrm{~mm} \mathrm{Hg}$ & 85.49 & 84.03 & \\
\hline $161-260 \mathrm{~mm} \mathrm{Hg}$ & 11.90 & 12.94 & \\
\hline Missing & 1.71 & 2.01 & \\
\hline Respiratory rate & & & 0.68 \\
\hline 4-11 breaths/min & 0.24 & 0.19 & \\
\hline 12-20 breaths/min & 87.88 & 86.40 & \\
\hline 21-60 breaths/min & 8.90 & 10.09 & \\
\hline Missing & 2.98 & 3.32 & \\
\hline \multicolumn{4}{|c|}{ Chief complaint associated with hospitalization } \\
\hline Chest pain and related symptoms & 7.37 & 6.40 & 0.48 \\
\hline Shortness of breath & 3.24 & 3.19 & 0.80 \\
\hline $\begin{array}{l}\text { Other symptoms/probably related } \\
\text { to psychological }\end{array}$ & 1.28 & 0.97 & 0.19 \\
\hline General weakness & 1.19 & 1.14 & 0.26 \\
\hline Labored or difficult breathing & 0.56 & 0.88 & 0.93 \\
\hline Fainting (syncope) & 0.44 & 0.42 & 0.09 \\
\hline Unconscious on arrival & 0.35 & 0.38 & 0.17 \\
\hline Other symptoms referable & 0.38 & 0.35 & 0.81 \\
\hline to the nervous system & & & \\
\hline
\end{tabular}

TABLE 2. Continued

\begin{tabular}{lccc}
\hline & $\begin{array}{c}\text { Hospitals With } \\
\text { Observation Units, } \\
\text { W\% }(\mathrm{N}=6,067)\end{array}$ & $\begin{array}{c}\text { Hospitals Without } \\
\text { Observation Units, } \\
\text { W\% }(\mathrm{N}=18,165)\end{array}$ & $P$ Value \\
\hline Chronic diseases & & & \\
Congestive heart failure & 4.13 & 4.05 & 0.05 \\
Cerebrovascular disease & 4.03 & 3.33 & 0.04 \\
Diabetes & 11.15 & 11.44 & 0.69 \\
HIV & 0.51 & 0.44 & 0.99 \\
On dialysis & 1.14 & 0.96 & 0.25 \\
\hline NOTE: Abbreviations: HIV, human immunodeficiency virus; W\%, weighted. & \\
\hline
\end{tabular}

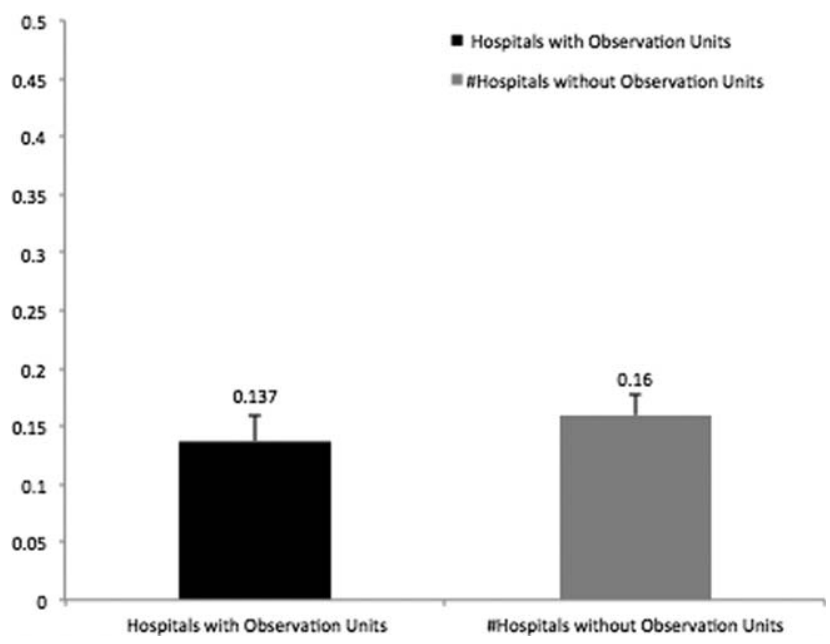

FIG. 1. Emergency department standardized admission rates for hospitals with and without observation units.

were unable to track hospitals longitudinally to determine if a hospital's high volume is associated with the creation of EDOUs as a means to offset its demand. However, in our analysis, we did control for overall patient volume when calculating the RHSAR. Finally, we were not able to limit the dataset to observation unit admission conditions because of the limited number of visits provided per hospital by NHAMCS. We conducted an analysis using $80 \%$ power and a $P$ value of 0.05 to determine the sample size needed to have statistically significant results. We would require 920 hospitals to have statistically significant results, which suggests we were underpowered to detect a statistically significant difference.

In this preliminary study, we did not find an association between the presence of EDOUs and ED hospital admissions. Our study was limited by an inability to analyze administrative differences and to adjust for certain hospital factors that are likely to influence inpatient admissions via the ED. Nonetheless, our findings suggest that EDOUs merit further evaluation of their potential cost savings and the quality of the care they provide. An evaluation of ED observation departmental management is also needed to assess differences in care at observation units managed by emergency physicians versus non-emergency physicians. 
Disclosures: R.C., B.S., and C.G. conceived the study. R.C. conducted the statistical analysis and was supervised by B.S. and C.G. All authors analyzed the results and interpreted findings. R.C. and D.B. drafted the manuscript, and all authors contributed substantially to its revision. All authors listed have contributed sufficiently to the project to be included as authors, and all those who are qualified to be authors are listed in the author byline. This work was previously presented at the 2013 Society for Academic Emergency Medicine Annual Meeting, Dallas, Texas. Dr. Capp is funded by a translational K award: KL2 TR001080. Dr. Gross reports grants from Johnson \& Johnson, Medtronic Inc., and 21st Century Oncology during the conduct of this study. In addition, he received payment from Fair Health Inc. and ASTRO outside the submitted work. Dr. Sun receives National Institutes of Health funding. No conflicts of interest, financial or other, exist. This applies to all authors.

\section{References}

1. Wiler JL, Ross MA, Ginde AA. National study of emergency department observation services. Acad Emerg Med. 2011;18(9):959-965.

2. Baugh CW, Venkatesh AK, Bohan JS. Emergency department observation units: a clinical and financial benefit for hospitals. Health Care Manage Rev. 2011;36(1):28-37.

3. Roberts RR, Zalenski RJ, Mensah EK, et al. Costs of an emergency department-based accelerated diagnostic protocol vs hospitalization in patients with chest pain: a randomized controlled trial. JAMA. 1997; 278(20):1670-1676.

4. Centers for Disease Control and Prevention. National Hospital Ambulatory Medical Care Survey. Ambulatory health care data. Questionnaires, datasets, and related documentation. 2009. Available at: http://www.cdc.gov/nchs/ahcd/ahcd_questionnaires.htm. Accessed November 1, 2011.

5. Capp R, Ross JS, Fox JP, et al. Hospital variation in risk-standardized hospital admission rates from US EDs among adults. Am J Emerg Med. 2014;32(8):837-843.

6. Venkatesh AK, Geisler BP, Gibson Chambers JJ, Baugh CW, Bohan JS, Schuur JD. Use of observation care in US emergency departments, 2001 to 2008. PloS One. 2011;6(9):e24326.

7. Baugh CW, Venkatesh AK, Hilton JA, Samuel PA, Schuur JD, Bohan JS. Making greater use of dedicated hospital observation units for many short-stay patients could save $\$ 3.1$ billion a year. Health Aff (Millwood). 2012;31(10):2314-2323.

8. Mace SE, Graff L, Mikhail M, Ross M. A national survey of observation units in the United States. Am J Emerg Med. 2003;21(7):529533.

9. Crenshaw LA, Lindsell CJ, Storrow AB, Lyons MS. An evaluation of emergency physician selection of observation unit patients. Am J Emerg Med. 2006;24(3):271-279.

10. Ross MA, Hockenberry JM, Mutter R, Barrett M, Wheatley M, Pitts SR. Protocol-driven emergency department observation units offer savings, shorter stays, and reduced admissions. Health Aff (Millwood). 2013;32(12):2149-2156. 